\section{Sulcal thickness as a vulnerability indicator}

\section{for schizophrenia}

\author{
VINA M. GOGHARI, KELLY REHM, CAMERON S. CARTER \\ and ANGUS W. MACDONALD
}

\begin{abstract}
Background People with
schizophrenia may demonstrate cortical

abnormalities, with gyri and sulci

potentially being differentially affected.
\end{abstract}

\begin{abstract}
Aims To measure frontal and temporal sulcal cortical thickness, surface area and volume in the non-psychotic relatives of patients with schizophrenia as a potential vulnerability indicator for the disorder.
\end{abstract}

\section{Method An automated parcellation} method was used to measure the superior frontal, inferior frontal, cingulate, superior temporal and inferior temporal sulci in the relatives of patients $(n=19)$ and controls $(n=22)$.

\section{Results Compared with controls, relatives had reversed hemispheric asymmetry in their cingulate sulcal thickness and a bilateral reduction in their superior temporal sulcal thickness.}

\section{Conclusions Cingulate and superior} temporal sulcal thickness abnormalities may reflect neural abnormalities associated with the genetic liability to schizophrenia. Cortical thinning in these regions suggests that liability genes affect the dendrites, synapses or myelination process during the neurodevelopment of the cortical mantle.

Declaration of interest None. Funding detailed in Acknowledgements.
Sulco-gyral patterns are thought to form through minimisation of axonal tension during cortico-cortical connection formation (Van Essen, 1997; Hilgetag \& Barbas, 2005). This process results in morphological differences in the gyri and sulci, which include the number and shape of cells. Early disruption of axonal tracts can affect later development of the cortical mantle. Assessing differences in the cortical integrity of sulci compared with gyri may index neurodevelopmental abnormalities in cortical development in schizophrenia. Recent advances in brain imaging methods now allow efficient measurement of both sulci and gyri, including cortical thickness (e.g. Fischl et al, 2004). Investigations of the cortical mantle have demonstrated thickness abnormalities in temporal gyri and in frontal, temporal and parietal sulci in children and adolescents with schizophrenia-spectrum disorders (White et al, 2003); increased right temporal cortical folding (Harris et $a l, 2004 a$ ) and reduced posterior cingulate folding in patients with schizophrenia (Wheeler \& Harper, 2007); and increased right frontal cortical folding in high-risk individuals who later developed schizophrenia (Harris et al, 2004b). Studying adult non-psychotic relatives of patients provides a way to investigate the potential effects of genes or gene-environment interactions not confounded by the illness process or medication. Family studies have found increased frontal cortical folding in unaffected relatives (Falkai et al, 2007) and reduced left frontal cortical folding in high-risk individuals (Jou et al, 2005). We previously investigated gyri in the same sample of non-psychotic relatives of people with schizophrenia and found abnormalities in the cingulate, superior temporal, middle temporal and parahippocampal regions (Goghari et al, 2007). To characterise further the potential effect of susceptibility genes on cortical folding, we evaluated sulcal thickness, surface area and grey-matter volume in frontal and temporal regions. This complementary focus on sulci allows a more complete understanding of the potential structural abnormalities of the cortical mantle related to the schizophrenia diathesis.

\section{METHOD}

\section{Participants}

Twenty-two first-degree relatives of patients with schizophrenia and 23 controls participated. First-degree relatives were recruited by first identifying probands receiving treatment through the Western Psychiatric Institute and Clinic in Pittsburgh, Pennsylvania. Diagnosis for probands was confirmed using the Structured Clinical Interview for DSM-IV (First et al, 1996). Control group participants were recruited from the general population. Individuals were excluded if they had experienced head trauma, seizures or had a diagnosis of substance misuse or dependence within the preceding 6 months. Control group candidates were excluded additionally if they had a family history of psychosis. Relatives and controls were screened for psychiatric symptoms or disorders using the Structured Clinical Interview for DSM-III-R, Nonpatient version (Spitzer et al, 1990). Relatives were additionally assessed with the Structured Interview for DSM-III Personality Disorders-Revised (SID-P) for cluster A personality disorders (Pfohl et al, 1982). One control met criteria for DSM-IV diagnosis in the past month (intermittent explosive disorder). Three relatives and three controls met criteria for lifetime diagnosis for major depressive disorder. In addition, one relative met criteria for schizotypal personality disorder. This protocol was approved by the University of Pittsburgh institutional review board. After complete description of the study to the participants, written informed consent was obtained.

\section{Image acquisition and processing}

Spoiled gradient recalled magnetic resonance imaging (MRI) scans were acquired in the axial plane using a GE Signa (General Electric, Milwaukee, Wisconsin, USA) $3 \mathrm{~T}$ magnetic resonance scanner $(0.9375 \mathrm{~mm} \times$ $0.9375 \mathrm{~mm} \times 1.5 \mathrm{~mm}$ voxel size, 124 slices). Whole-brain volumes were extracted using McStrip, an automated, consensus-based stripping algorithm (Rehm et al, 2004). Whole-brain volumes were intensitycorrected using non-parametric nonuniform intensity normalisation (N3) to improve the accuracy of tissue classification 
and cortical surface extraction (Sled et al, 1998). An automatic algorithm labelled the left and right hemispheres, cerebellum and brain-stem (Rehm et al, 2005). Defects were manually edited where necessary. These stripped, intensity-corrected brain images and grossly subdivided volumes were used in the subsequent steps.

Surface extraction and cortical parcellation were conducted using FreeSurfer version 1.3 (Fischl et al, 2004) (DS1, see data supplement to online version of this paper). Briefly, the stripped, intensity-corrected, subdivided volume was segmented to classify white matter and to approximate the grey-white matter boundary for each cortical hemisphere, from which a topologically correct grey-white matter boundary surface triangulation was generated (Dale et al, 1999; Fischl et al, 2001). Subsequently, a pial surface was generated using a deformable surface algorithm (Fischl \& Dale, 2000). All surfaces were visually inspected and defects leading to major topological errors were manually corrected. The greywhite boundary surface was inflated and individual differences in curvature were normalised. For each brain examined the inflated surface was morphed into a sphere and registered to an average spherical surface that optimally aligned sulci and gyri across participants (Fischl et al, 1999a,b). Surfaces were successfully extracted for 41 participants (22 controls and 19 relatives) using standard parameters.

Parcellation for each individual's left and right hemisphere surface was transmitted from the parcellation of the reference spherical surface to which they were aligned. Each parcellated region was mapped back onto each individual brain's inflated surface by inverting the algorithm that morphed the inflated surface to the average spherical surface representation (Kuperberg et al, 2003; Fischl et al, 2004). Eighty-five parcellation units were provided by FreeSurfer, based on the conventions of Duvernoy (1991).

Thicknesses were calculated using FreeSurfer software; they were computed for each vertex in the triangulated surfaces by finding the point on the white-matter surface that was closest to a given point of the pial surface (and vice versa) and the average was taken between these two values. Surface areas were calculated for both the white-grey boundary and pial surface using the formula of Heron (Gellert et al, 1975). The average of the two surface areas at each triangle was computed and was used in analyses to simulate an intermediate cortical surface. Grey-matter volumes were calculated by constructing a closed mesh joining a pair of linked triangles and computing the enclosed volume (Eberly et al, 1991). Individual triangle volumes were aggregated to compute cortical grey-matter volume for each region of interest. This methodology has been extensively validated (Kuperberg et al, 2003; Fischl et al, 2004). A trained rater manually traced regions of interest on ten white-grey boundary inflated surfaces. An index of similarity between the automated and hand-drawn regions was calculated. This index was defined as the ratio of twice the common area between the two methods relative to the sum of the individual areas; a value above 0.7 was considered excellent correspondence (Zijdenbos et al, 1994). All three sulcal regions had acceptable correspondence (superior frontal 0.71, cingulate 0.82 , superior temporal, 0.87 ).

\section{Analyses}

All sulcal regions of interest were assessed using mixed-model analysis of covariance (ANCOVA), with hemisphere (left, right) entered as an intra-individual effect and group (control, relative) entered as an inter-individual effect. Age, gender and average cortical thickness were entered as covariates when analysing regional thicknesses; age, gender and total cortical surface area were entered as covariates when analysing regional surface areas; and age, gender and intracranial volume were entered as covariates when analysing regional volumes. All analyses were also conducted with education as an additional covariate; as this did not affect the results, the findings reported below do not include it as a covariate. Greenhouse-Geisser correction is reported for the mixed-model ANCOVAs. These planned comparisons were set to a

Table I Demographic characteristics of the sample

\begin{tabular}{lcccc}
\hline & $\begin{array}{c}\text { Control group } \\
(n=22)\end{array}$ & $\begin{array}{c}\text { Relatives group } \\
(n=19)\end{array}$ & Test statistic & $P$ \\
\hline Age, years: mean (s.d.) & $34.1(8.4)$ & $34.2(I I)$ & $t=0.02$ & 0.98 \\
Male gender, $n$ (\%) & $10(46)$ & $8(42)$ & $\chi^{2}=0.05$ & 0.83 \\
Minority ethnicity, $n$ (\%)' & $6(27)$ & $9(47)$ & $\chi^{2}=1.78$ & 0.18 \\
Education, years: mean (s.d.) & & & & 0.08 \\
$\quad$ Participants & $15.7(1.9)$ & $14.2(3.1)$ & $t=-1.79$ & 0.50 \\
Father's education & $13(2.8)$ & $13.6(2.6)$ & $t=0.68$ & 0.22 \\
Mother's education & $13.1(2.2)$ & $14.1(2.7)$ & $t=1.24$ & \\
\hline
\end{tabular}

I. African American. significance threshold of $P=0.05$. Because of concerns related to multiple comparisons, effect sizes are reported as a supplemental indicator to significance values. Partial etasquared effect sizes are presented: 0.01 is considered a small effect, 0.06 is considered a medium effect and 0.14 is considered a large effect (Stevens, 2002).

\section{RESULTS}

Data for the final sample are presented in Table 1. No significant difference between groups was found for any demographic variable. Although relatives tended to have slightly less educational attainment than the controls, parents were matched for education. The imaging data from three relatives and one control had to be excluded owing to poor quality. As one relative met criteria for schizotypal personality disorder, all analyses were conducted both with and without this participant: none of the findings changed substantially when this participant's data were removed.

\section{MRI analyses}

\section{Frontal sulcal measures}

Planned comparisons of middle and inferior frontal sulcal thickness, surface area and volume revealed no significant main effect of group or hemisphere by group interactions (all $P>0.2$ ).

\section{Cingulate sulcal measures}

A significant hemisphere by group interaction was found in cingulate sulcal thickness $(F=5.50, \quad$ d.f. $=1,36, \quad P=0.03 ; \quad$ partial $\eta^{2}=0.13$ ), with the relatives group having a reversal in hemispheric asymmetry pattern compared with the control group. The control group had thicker sulci in the left hemisphere, whereas those in the relatives group had thicker sulci in the right 
hemisphere (Fig. 1, Table 2). There were also significant effects of age $(F=7.26$, d.f. $=1,36, P=0.01)$ and average cortical thickness $(F=23.85$, d.f. $=1,36, P<0.001)$ covariates. No significant difference was found in surface area or volume (all $P>0.1$ ).

\section{Temporal sulcal measures}

A significant effect of group was found in the superior temporal sulcal thickness $(F=8.17, \quad$ d.f. $=1,36, \quad P=0.007$; partial $\eta^{2}=0.19$ ) with the relatives group having $2.4 \%$ bilateral decrease (Fig. 1, Table 2). There was also a significant effect of average cortical thickness $(F=119.87$, d.f. $=$ $1,36, P<0.001)$ covariate. No significant difference was found in surface area or volume (all $P>0.1$ ). Planned comparisons of inferior temporal sulci thickness, surface area and volume revealed no significant main effect of group or hemisphere by group interactions $(P>0.1)$.

\section{DISCUSSION}

This study evaluated whether frontal and temporal sulcal abnormalities were associated with a possible genetic vulnerability to schizophrenia. To examine possible grey-matter abnormalities comprehensively we measured cortical thickness, surface area and volume. The principal findings were that non-psychotic relatives of patients with schizophrenia demonstrated abnormalities in thickness in the cingulate and superior temporal sulci.

Consistent with previous findings of cingulate cortex abnormalities, we found that cingulate sulcal thickness was abnormal among the relatives, who were observed to have a reversed asymmetry pattern with the right hemisphere being thicker than the left. Similar to our findings, studies have demonstrated that controls had more leftward asymmetry in their paracingulate sulci compared with patients with schizophrenia (Yücel et al, 2002; Le Provost et $a l, 2003)$, whereas the patients with schizophrenia had more rightward asymmetry in their paracingulate sulci (Le Provost et al, 2003). Compared with a control group, individuals with a high risk of developing schizophrenia tended to have a significantly more interrupted left cingulate sulcus and less well-developed left paracingulate sulcus (Yücel et al, 2003). Recent MRI studies

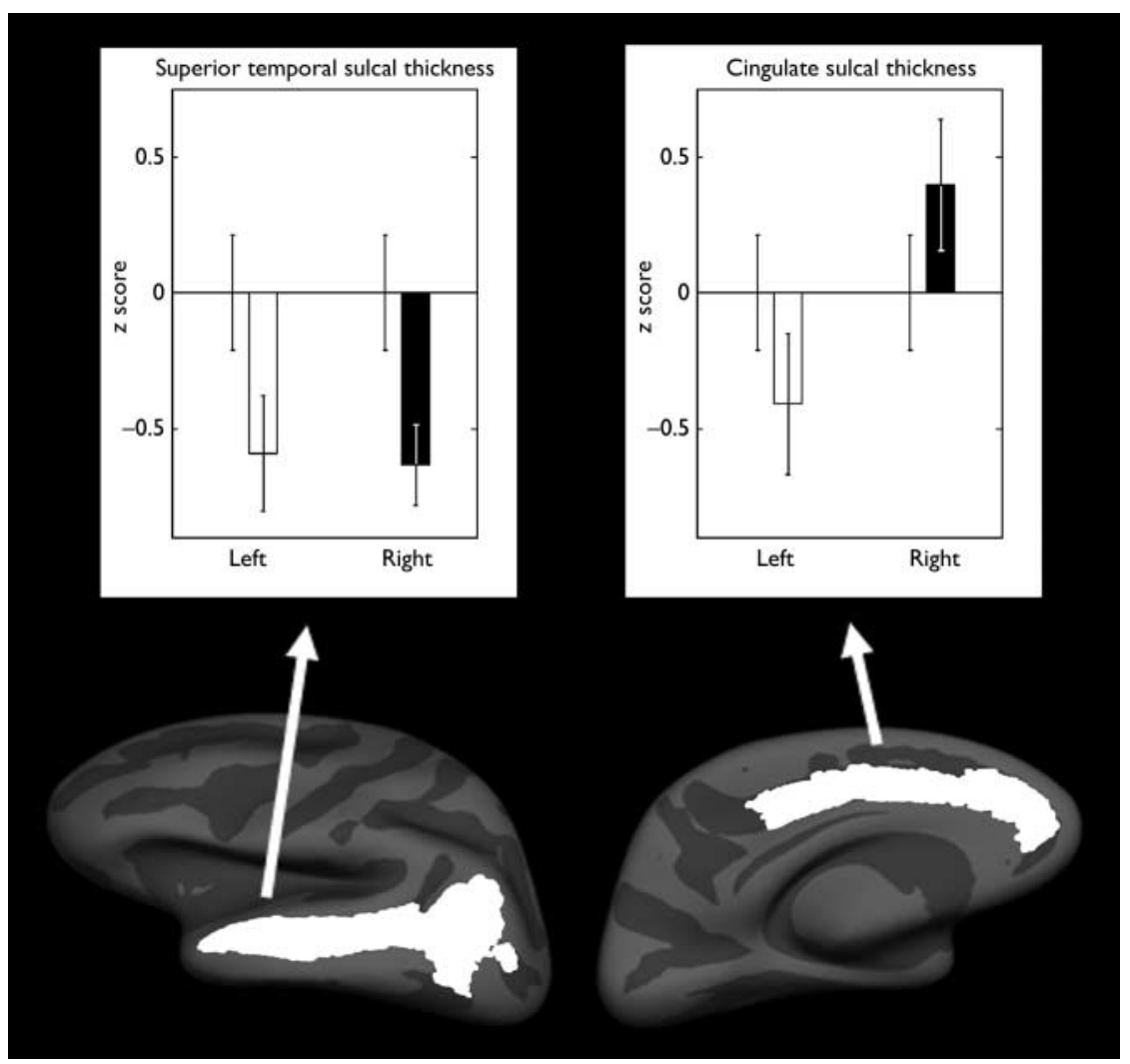

Fig. I Mean z scores for the thicknesses that demonstrated a significant difference between the control group $(n=22)$ and the relatives group $(n=19)$. Left-hand panel, superior temporal sulcal thickness; right-hand panel, cingulate sulcal thickness. Mean scores for the relatives group for the left and right hemispheres are compared with the control group score (mean $=0$ ); bars indicate standard error of the mean. of cortical thickness have found the cingulate cortex to be thinner in people with first-episode schizophrenia (Narr et al, 2005) and specifically the anterior cingulate gyri to be thinner in patients with chronic schizophrenia (Kuperberg et al, 2003). A study assessing the gyrification index, defined as the ratio of the length of the contour of the gyri and sulci compared with the length connecting the gyral surface, demonstrated significant reductions in posterior cingulate cortical folding in schizophrenia (Wheeler \& Harper, 2007). Consistent with neuroimaging results of cortical thinning, post-mortem work on the anterior cingulate cortex of people with schizophrenia has demonstrated glial cell loss and changes in cell size and density (Benes et al, 1991, 2001; Stark et al, 2004). However, a greater number of axons in layer II and sub-lamina IIIA has also been reported (Benes et al, 1986). The cingulate gyri in the current sample have also been noted to be thinner and have reduced surface area and volume in the relatives group (Goghari et al, 2007). Taken together these results suggest neurodevelopmental abnormalities in this limbic region, which has an essential role in affective, cognitive and motor control systems (Vogt et al, 1992).

Consistent with temporal cortex abnormalities reported in the literature, we found a bilateral reduction in thickness in the superior temporal sulci in the non-psychotic relatives of patients with schizophrenia compared with controls. Consistent with this finding, the cell density in a variety of temporal lobe sulci was reduced in schizophrenia (Chance et al, 2004). A study of male patients with chronic schizophrenia demonstrated faster cerebrospinal fluid volume expansion in posterior superior temporal sulci compared with controls over a span of 4 years (Mathalon et al, 2001). The superior temporal sulci border both the superior and middle temporal gyri. Previously in the current sample, only surface area and not thickness was decreased in the superior temporal gyri (Goghari et al, 2007). In addition, the left middle temporal and bilateral parahippocampal gyri were greater in surface area and volume in the relatives group. Superior temporal lobe gyrus volume has also been shown to be reduced in the young offspring of patients (Rajarethinam et al, 2004). Together, these findings suggest that the temporal sulci and gyri are globally affected. Thus, the temporal cortex may be an important indicator of the schizophrenia diathesis. 
Table 2 Thickness, surface area and grey-matter volume (raw values; mean, s.d.)

\begin{tabular}{|c|c|c|c|c|c|c|}
\hline & \multicolumn{2}{|c|}{ Thickness (mm) } & \multicolumn{2}{|c|}{ Surface area $\left(\mathrm{mm}^{2}\right)$} & \multicolumn{2}{|c|}{ Volume $\left(\mathrm{mm}^{3}\right)$} \\
\hline & Controls & Relatives & Controls & Relatives & Controls & Relatives \\
\hline \multicolumn{7}{|l|}{ Frontal sulci } \\
\hline Left superior frontal & $2.54(0.17)$ & $2.56(0.2 I)$ & 2697 (409) & $2538(322)$ & $688 \mathrm{I}(976)$ & $6578(1095)$ \\
\hline Right superior frontal & $2.56(0.17)$ & $2.57(0.19)$ & $2165(340)$ & $2090(363)$ & $5558(1034)$ & $5410(1137)$ \\
\hline Left inferior frontal & $2.42(0.18)$ & $2.37(0.22)$ & $1828(308)$ & $1785(2 \mid 7)$ & $4372(808)$ & $4179(723)$ \\
\hline Right inferior frontal & $2.40(0.15)$ & $2.36(0.19)$ & $1544(312)$ & $1539(301)$ & $3653(795)$ & $3589(68 I)$ \\
\hline \multicolumn{7}{|l|}{ Cingulate sulci } \\
\hline Left cingulate & $2.71(0.14)$ & $2.67(0.22)$ & $2497(294)$ & $2405(375)$ & $6751(893)$ & $6378(1122)$ \\
\hline Right cingulate & $2.67(0.14)$ & $2.70(0.19)$ & $2672(379)$ & $2725(396)$ & $7099(1217)$ & $7320(1076)$ \\
\hline \multicolumn{7}{|l|}{ Temporal sulci } \\
\hline Left superior temporal & $2.65(0.15)$ & $2.58(0.17)$ & $4312(369)$ & $4109(513)$ & II299 (1239) & $10480(1542)$ \\
\hline Right superior temporal & $2.47(0.18)$ & $2.42(0.21)$ & $4609(546)$ & $4626(691)$ & III 85 (I50I) & $10927(1678)$ \\
\hline Left inferior temporal & $2.64(0.21)$ & $2.60(0.15)$ & $1043(273)$ & II 24 (202) & $2653(620)$ & $2856(557)$ \\
\hline Right inferior temporal & $2.56(0.21)$ & $2.59(0.25)$ & $900(233)$ & $860(183)$ & $2232(585)$ & $2|4|(4 \mid 5)$ \\
\hline
\end{tabular}

We failed to find any significant structural differences in the middle and inferior frontal regions, although functional MRI data collected on the same sample of relatives and controls suggest abnormalities in prefrontal activity (Brodmann areas 9, 8 and 6) (MacDonald et al, 2006). In contrast to our structural findings, Falkai et al (2007) conducted a gyrification study of patients with schizophrenia and unaffected relatives and found both groups to have a higher frontal gyrification index than a control group. Findings have been inconsistent, however, with one study finding that high-risk individuals who later developed schizophrenia had a higher gyrification index in the right frontal lobe (Harris et al, 2004b), and another study finding that high-risk individuals had no difference in the right frontal lobe, but rather a reduced gyrification index in the left frontal lobe (Jou et al, 2005). These discrepancies in findings may be sample-specific or due to methodological differences. Our sample size is modest and might not have been sufficient to detect smaller group differences. However, in our sample, the effect sizes indicated the magnitude of the difference between the groups was small: superior and inferior frontal sulcal thickness, partial $\eta^{2}=0-0.06$; surface area, partial $\eta^{2}=0.01-$ 0.03 ; volume, partial $\eta^{2}=0-0.01$.

\section{Limitations}

We attempted to control for multiple comparisons by restricting our analyses to five planned comparisons of regions consistently associated with schizophrenia. There is a need for caution when interpreting results that derive from multiple comparisons. However, this caution has to be balanced by the need to be sensitive to group differences. Our approach to this balance was to report effect sizes, which reflect the magnitude of the difference between groups regardless of sample size. Our results indicated the abnormality in the cingulate sulcal thickness (effect size 0.13) and the bilateral reduction in superior temporal sulci (effect size 0.19 ) are both noteworthy findings. This study compared the non-psychotic relatives of patients with schizophrenia and controls. It would have been useful in addition to have collected patient pedigree information to quantify genetic liability on a continuum. We used an automated method to measure cortical thickness and to parcellate the cerebral cortex. The accuracy of the thickness values depends on the accuracy of the grey-white segmentation and therefore can be influenced by various artefacts. However, we followed validated data processing procedures provided by the FreeSurfer manual and all analyses were performed in consultation with a magnetic resonance physicist (K.R.).

\section{Implications}

Sulci and gyri are thought to form together, with gyri forming between densely connected regions and sulci forming between weakly connected regions (Hilgetag \& Barbas, 2006). In addition, structural differences exist between the gyri and sulci, with gyri having significantly greater number of neurons in deep layers. The process of cortical folding can lead to differences in cell and dendrite morphology and the layout of cortical blood vessels in the gyri and sulci, thereby potentially resulting in further differences in functioning (Hilgetag \& Barbas, 2005). Assessing gyri and sulci may reflect a further way to index neurodevelopmental abnormalities of cortical development in schizophrenia.

In this study we found the cingulate and the superior temporal sulci to be the most affected, and these may serve as useful structural endophenotypes relating the pathology to the aetiology. In addition, our results indicate that thickness, surface area and volume are differentially affected in the sulci and gyri. Combined with our previous gyral findings, these sulcal results suggest that cortical thickness abnormalities are more prominent in the sulci and that surface area abnormalities are more prominent in the gyri. A number of factors could change the formation of sulci and gyri. Differential growth of cortical regions could displace adjacent gyral regions. Alternatively, growth of brain regions could affect the trajectories of migrating axons, affecting sulci and gyri formation patterns. Lastly, time course disruption of interhemispheric and intercortical white-matter tracts, especially of the thalamocortical fibre system, might affect sulci and gyri formation patterns, even in distant areas (Welker, 1990). The schizophrenia diathesis most likely has an impact on all these processes, thereby having a complex effect on the neurodevelopment of cortical topography. 


\section{ACKNOWLEDGEMENTS}

V.M.G. was supported by a Postgraduate Scholarship Master's and Doctoral Award from the Natural Sciences and Engineering Research Council of Canada and by the Graduate Research Partnership Program, University of Minnesota. Additional support was provided by the National Institute of Mental Health (MH45156) and the University of Minnesota. The authors gratefully acknowledge the assistance of the International Neuroimaging Consortium: Dr David Rottenberg, Kirt Schaper, Kristi Boesen and Tim Jarvis; the University of Pittsburgh Medical Center, Department of Radiology; the Cognitive Control Neuroscience Laboratory; the Western Psychiatric Institute and Clinic, University of Pittsburgh; and Theresa Becker, jill Stanton, Ryan Walter, and Bruce Fischl. We especially thank James Porter for his help with reliability analyses.

\section{REFERENCES}

Benes, F. M., Davidson, J. \& Bird, E. D. (1986)

Quantitative cytoarchitectural studies of the cerebra cortex of schizophrenics. Archives of General Psychiatry, 43, $31-35$

Benes, F. M., McSparren, J., Bird, E. D., et al (1991) Deficits in small interneurons in prefrontal and cingulate cortices of schizophrenic and schizoaffective patients. Archives of General Psychiatry, 48, 996-100I.

Benes, F. M., Vincent, S. L. \& Todtenkopf, M. (200I) The density of pyramidal and nonpyramidal neurons in anterior cingulate cortex of schizophrenic and bipolar subjects. Biological Psychiatry, 50, 395-406.

Chance, S. A., Tzotzoli, P. M., Vitelli, A., et al (2004) The cytoarchitecture of sulcal folding in Heschl's sulcus and the temporal cortex in the normal brain and schizophrenia: lamina thickness and cell density. Neuroscience Letters, 367, 384-388.

Dale, A. M., Fischl, B. \& Sereno, M. I. (1999) Cortical surface-based analysis. I. Segmentation and surface reconstruction. Neuroimage, 9, 179-194.

Duvernoy, D. M. (199I) The Human Brain: Surface, Three-Dimensional Sectional Anatomy and MRI. Springer.

Eberly, D., Lancaster, J. \& Alyassin, A. (199I) On gray scale image measurements: Il. Surface area and volume. CVGIP: Graphical Models and Image Processing, 53. $550-562$

Falkai, P., Honer, W. G., Kamer, T., et al (2007) Disturbed frontal gyrification within families affected with schizophrenia. Journal of Psychiatric Research, 4I 805-813.

First, M. B., Spitzer, R. L., Gibbon, M., et al (1996) Structured Clinical Interview for DSM-IV Axis I Disorders Patient Version (SCID-P, Version 2). New York State Psychiatric Institute, Biometrics Research.

Fischl, B. \& Dale, A. M. (2000) Measuring the thickness of the human cerebral cortex from magnetic resonance images. Proceedings of the National Academy of Sciences of the United States of America, 97, II05011055.

\section{Fischl, B., Sereno, M. I. \& Dale, A. M. (1999a)}

Cortical surface-based analysis. II: Inflation, flattening, and a surface-based coordinate system. Neuroimage, 195-207.

Fischl, B., Sereno, M. I., Tootell, R. B., et al (1999b) High-resolution intersubject averaging and a coordinate system for the cortical surface. Human Brain Mapping, 8 $272-284$

VINA M. GOGHARI, MA, Department of Psychology, University of Minnesota; KELLY REHM, PhD, Department of Radiology, University of Minnesota, Twin Cities, Minnesota; CAMERON S. CARTER, MD, Imaging Research Center, University of California, Davis, California; ANGUS W. MACDONALD, PhD, Department of Psychology, University of Minnesota, Twin Cities, Minnesota, USA

Correspondence: Angus W. MacDonald, III, Department of Psychology, University of Minnesota, N218 Elliott Hall, 75 East River Road, Minneapolis, MN 55455, USA. Tel: + I 612 624 38I3; fax: + I 612625 6668; email: angus@umn.edu

(First received 29 January 2007, final revision 29 April 2007, accepted 9 May 2007)

Fischl, B., Liu, A. \& Dale, A. M. (200I) Automated manifold surgery: constructing geometrically accurate and topologically correct models of the human cerebral cortex. IEEE Transactions on Medical Imaging, 20, 70-80.

Fischl, B., van der Kouwe, A., Destrieux, C., et al (2004) Automatically parcellating the human cerebral cortex. Cerebral Cortex, 14, II-22.

Gellert, W., Kustner, H., Hellwich, M., et al (1975) The VNR Concise Encyclopedia of Mathematics. Van Nostrand Reinhold.

Goghari, V. M., Rehm, K., Carter, C. S., et al (2007) Regionally specific cortical thinning and gray matter abnormalities in the healthy relatives of schizophrenia patients. Cerebral Cortex, I7, 415-424.

Harris, J. M., Yates, S., Miller, P., et al (2004a) Gyrification in first-episode schizophrenia: a morphometric study. Biological Psychiatry, 55, 141-147.

Harris, J. M., Whalley, H., Yates, S., et al (2004b) Abnormal cortical folding in high-risk individuals: a predictor of the development of schizophrenia? Biological Psychiatry, 56, 182-189.

Hilgetag, C. C. \& Barbas, H. (2005) Developmenta mechanics of the primate cerebral cortex. Anatomy and Embryology, 210, 4II-417.

Hilgetag, C. C. \& Barbas, H. (2006) Role of mechanical factors in the morphology of the primate cerebral cortex. PLoS Computational Biology, 2, e22.

Jou, R. J., Hardan, A. Y. \& Keshavan, M. S. (2005) Reduced cortical folding in individuals at high risk for schizophrenia: a pilot study. Schizophrenia Research, 75 309-313.

Kuperberg, G. R., Broome, M. R., McGuire, P. K. et al (2003) Regionally localized thinning of the cerebral cortex in schizophrenia. Archives of General Psychiatry, 60, 878-888.

Le Provost, J. B., Bartrés-Faz, D., PaillèreMartinot, M. L., et al (2003) Paracingulate sulcus morphology in men with early-onset schizophrenia British Journal of Psychiatry, 182, 228-232.

MacDonald, A.W., Becker, T. M. \& Carter, C. S. (2006) Functional magnetic resonance imaging study of cognitive control in the healthy relatives of schizophrenia patients. Biological Psychiatry, 60, 1241-1249.

Mathalon, D. H., Sullivan, E.V., Lim, K. O., et al (200I) Progressive brain volume changes and the clinica course of schizophrenia in men: a longitudinal magnetic resonance imaging study. Archives of General Psychiatry 58, 148-157.

Narr, K. L., Toga, A. W., Szeszko, P., et al (2005) Cortical thinning in cingulate and occipital cortices in first episode schizophrenia. Biological Psychiatry, 58, 32-40.

Pfohl, B., Stagl, D. \& Zimmerman, M. (1982) The Structured Interview for DSM-III Personality Disorders (SID-P). University of lowa Department of Psychiatry.

Rajarethinam, R., Sahni, S., Rosenberg, D. R., et a (2004) Reduced superior temporal gyrus volume in young offspring of patients with schizophrenia. American Journal of Psychiatry, 161, ||2|-||24.

Rehm, K., Schaper, K., Anderson, J., et al (2004) Putting our heads together: a consensus approach to brain/non-brain segmentation in TI-weighted MR volumes. Neuroimage, 22, 1262-1270.

Rehm, K., Goghari, V. M. \& Rottenberg, D. A. (2005) Automatic extraction of left and right hemispheres from MRI brain volumes using a refined registration method. Neuroimage, 26 (suppl. I), CD-ROM.

Sled, J. G., Zijdenbos, A. P. \& Evans, A. C. (1998) A nonparametric method for automatic correction of intensity nonuniformity in MRI data. IEEE Transactions on Medical Imaging, 17, 87-97.

Spitzer, R. L., Williams, J. B. W., Gibbon, M., et al (1990) Structured Clinical Interview for DSM-III-RNon-Patient Edition (SCID-NP, Version I.0). American Psychiatric Press.

Stark, A. K., Uylings, H. B., Sanz-Arigita, E., et al (2004) Glial cell loss in the anterior cingulate cortex, a subregion of the prefrontal cortex, in subjects with schizophrenia. American Journal of Psychiatry, I6I, 882-888.

Stevens, J. P. (2002) Applied Multivariate Statistics for the Social Sciences (4th edn). Erlbaum.

Van Essen, D. C. (1997) A tension-based theory of morphogenesis and compact wiring in the central nervous system. Nature, 385, 313-318.

Vogt, B. A., Finch, D. M. \& Olson, C. R. (1992) Functional heterogeneity in cingulate cortex: the anterior executive and posterior evaluative regions. Cerebral Cortex, 2, 435-443.

Welker, W. (1990) Why does the cerebral cortex fissure and fold? A review of determinents of gyri and sulci. In Cerebral Cortex (eds E. G. Jones \& A. Peters), pp. 3-136. Plenum.

Wheeler, D. G. \& Harper, C. G. (2007) Localised reductions in gyrification in the posterior cingulate: schizophrenia and controls. Progress in NeuroPsychopharmacology and Biological Psychiatry, 31, 319-327.

White, T., Andreasen, N. C., Nopoulos, P., et al (2003) Gyrification abnormalities in childhood- and adolescent-onset schizophrenia. Biological Psychiatry, 54, $418-426$

Yücel, M., Stuart, G. W., Maruff, P., et al (2002) Paracingulate morphologic differences in males with established schizophrenia: a magnetic resonance imaging morphometric study. Biological Psychiatry, 52, 15-23.

Yücel, M., Wood, S. J., Phillips, L. J., et al (2003) Morphology of the anterior cingulate cortex in young men at ultra-high risk of developing a psychotic illness. British Journal of Psychiatry, 182, 518-524

Zijdenbos, A. P., Dawant, B. M., Margolin, R. A., et al (1994) Morphometric analysis of white matter lesions in MR images: method and validation. IEEE Transactions on Medical Imaging, 13, 716-724. 